Noname manuscript No.

(will be inserted by the editor)

\section{First Steps Toward Translating Robotic Walking To Prostheses: A Nonlinear Optimization Based Control Approach}

\author{
Huihua Zhao · Jonathan \\ Horn · Jake Reher . \\ Victor Paredes · Aaron D. \\ Ames
}

Received: date / Accepted: date

\begin{abstract}
This paper presents the first steps toward successfully translating nonlinear real-time optimization based controllers from bipedal walking robots to a self-contained powered transfemoral prosthesis: AMPRO, with the goal of improving both the tracking performance and the energy efficiency of prostheses control. To achieve this goal, a novel optimal control strategy combining control Lyapunov function (CLF) based quadratic programs (QP) with impedance control is proposed. This optimal controller is first verified on a human-like bipedal robot platform, AMBER. The results indicate improved (compared to variable impedance control) tracking performance, stability and robustness to unknown disturbances. To translate this complete methodology to a prosthetic device with an amputee, we begin by collecting reference human locomotion data via Inertial measurement Units (IMUs). This data forms the basis for an optimization problem that generates virtual constraints, i.e., parameterized trajectories, specifically for the amputee and the prosthesis. A online optimization based controller is uti-
\end{abstract}

Huihua Zhao

Mechanical Engineering, Georgia Institute of Technology

Atlanta, GA USA. E-mail: huihua@gatech.edu

Jonathan Horn

Mechanical Engineering, Texas A\&M University

College Station, TX USA. E-mail: j.horn@tamu.edu

Jacob Reher

Mechanical Engineering, Georgia Institute of Technology

Atlanta, GA USA. E-mail: jpreher@gatech.edu

Victor Paredes

Mechanical Engineering, Texas A\&M University

College Station, TX USA. E-mail: vcparedesc@tamu.edu

Aaron D. Ames

Mechanical Engineering, Georgia Institute of Technology

Atlanta, GA USA. E-mail: ames@gatech.edu lized to optimally track the resulting desired trajectories.

The parameterization of the trajectories is determined through a combination of on-board sensing on the prosthesis together with IMU data, thereby coupling the actions of the user with the controller. Importantly, the proposed control law displays remarkable tracking and improved energy efficiency, outperforming PD and impedance control strategies. This is demonstrated experimentally on the prosthesis AMPRO through the implementation of the holistic sensing, algorithm and control framework, with the end result being stable prosthetic walking by an amputee.

Keywords Transfemoral prosthesis control · real-time optimal control · hybrid systems - quadratic program . optimization problem

\section{INTRODUCTION}

There are approximately 222,000 people in the United States alone that are transfemoral amputees [1]. Despite this large amputee population, the current market for commercial transfemoral prostheses remains largely limited to energetically passive prosthetic devices, limiting the day-to-day life of amputees with increased metabolic cost and constrained locomotion capabilities [2]. As one of the most important applications of bipedal robotic research, powered lower-limb prostheses capable of providing net power in conjunction with various prostheses controllers have been developed in recent decades. Most notably, [3,4] developed a hydraulically actuated knee prosthesis with the "echo control" method to mirror the modified trajectory of a healthy leg to the opposing side. Control based on gait-pattern generators has been realized in $[5,6]$. An optimal design of a transfemoral prosthesis with energy storage and regeneration is presented in [7]. Using the Center of Pressure (COP) as the phase variable, virtual constraint control of a powered prosthetic leg has been realized in [8]. Under the assumption that the human gait is cyclic, variable impedance control is one of the most common approaches for controlling prostheses [9-14]. However, despite the improvements that these smart controllers have achieved, there are still limitations related to the optimality of the controllers and the need for exhaustive clinical testing to determine control parameters. These issues motivate the main objectives of this paper.

The primary goal of this paper is to demonstrate the successful translation of advanced (optimal) controllers from robotic walking to prostheses, with the end result being improved energy efficiency given similar tracking performance compared to existing control strategies (PD and variable impedance control). In particular, the main contributions of this work are threefold: a) to propose the idea of using bipedal robots to test prosthetic controllers. A nominal walking gait is found for the robot platform which displays qualitatively 
human-like walking, and prosthetic controllers are tested on one "leg" of the robot. Through this method, we are able to present and test a online optimization based transfemoral prosthesis control method: control Lyapunov function (CLF) based quadratic programs (QPs) coupled with variable impedance control. b) To introduce the custom designed selfcontained powered transfemoral prosthetic device AMPRO explicitly with the goal to validate this systematic method. c) Having verified the controllers on the robot platform, we translate the complete methodology from realizing humanlike robotic walking to achieve stable prosthetic walking on the transfemoral prosthesis device: AMPRO.

The goals of this paper are motivated by the shortcomings (hand tuning and passiveness) of impedance control, a novel prosthetic controller that combines the rapidly exponentially stabilizing control Lyapunov functions (RESCLFs) with impedance control is proposed with the goal of achieving better tracking and improved energy efficiency on prostheses. This controller was first verified in simulation [15] and then tested on a human-like bipedal robot platform: AMBER, which has been used in previous work [16] to achieve stable "prosthetic" walking. These successes on robotic systems motivate the realization of this controller on a custom-built prosthetic device: AMPRO. We begin with utilizing a custom motion capture system consisting of Inertial Measurement Units (IMUs) to collect human locomotion trajectories. With the collected data, a human-inspired optimization problem is then leveraged to obtain a stable and robust gait for a specific test subject. IMUs are also used to estimate human movements during walking, thus providing human-robot sensory feedback.

Through the systematic methodology for translating human-inspired robotic walking to prostheses, stable prosthetic walking is realized for an amputee subject, as shown in Fig. 1. The powered prosthetic walking gait is compared with the original walking gait of the amputee subject using a passive device, showing that the powered gait is natural and human-like. More importantly, the proposed controller also outperforms other existing controllers (such as PD) w.r.t. both better tracking (21.6\% improvement on the $e_{r m s}$ ) and improved power consumption (5\% reduction). To summarize, the procedure for testing this controller both in simulation and on the bipedal robot helped to predict and resolve many implementation issues before attempting to realize walking with a human test subject. The presented procedure, therefore, has the potential to reduce the cost of clinical testing of prostheses through the fast and efficient development and testing of controllers.

The structure of this paper is as follows: In Sec. 2, human locomotion data collection with IMUs is introduced. Based on the collected data, human-inspired optimization is utilized to obtain the optimized trajectories for the bipedal robot or the prosthesis. Unifying RES-CLFs based quadratic
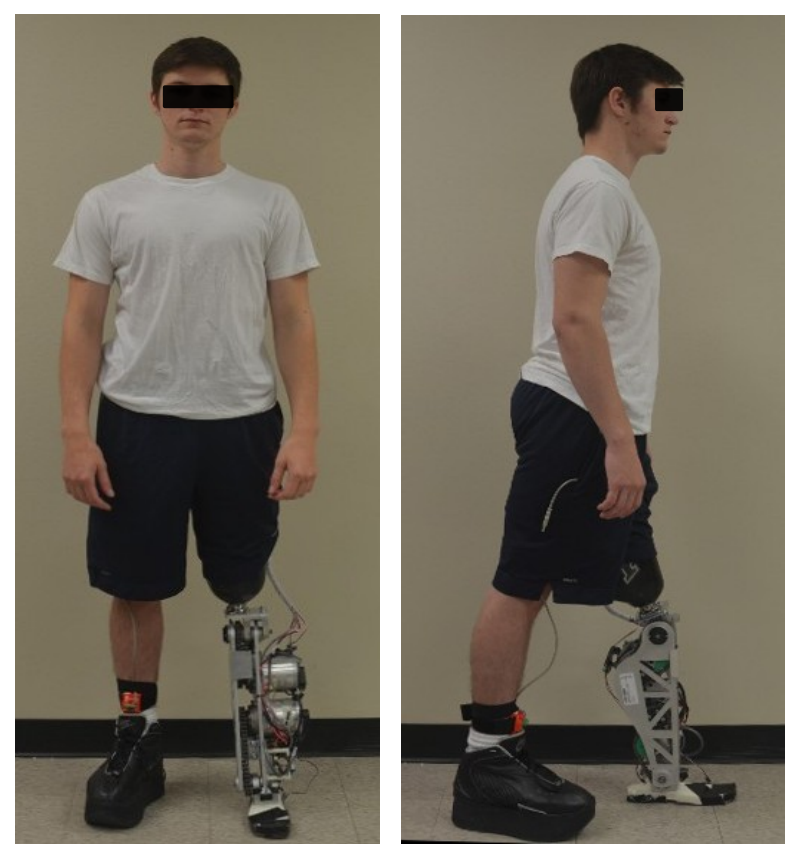

Fig. 1: Transfemoral test subject wearing the custom built prosthetic device, AMPRO.

programming control and the impedance control, the novel model independent quadratic program (MIQP) controller is discussed explicitly in Sec. 3. Before the implementation on the actual prosthetic device, the systematic methodology, including gait generation and optimal control, is verified in both simulation and experiment with the bipedal robot AMBER in Sec. 4. Finally, the experimental realization of the nonlinear online optimization based controller on a real prosthetic device with an amputee test subject is illustrated in Sec. 5. Conclusions and future work are presented at the end.

\section{ROBOTIC MODEL OF HUMAN LOCOMOTION}

In an effort to achieve human-like robotic walking, we turn to the most prevalent source for natural and efficient locomotion - the human body - for inspiration. In particular, a lowcost inertial motion capture system with IMUs is developed and interfaced with the human-inspired control approach. This system is first used to capture the walking trajectories of a human subject. Utilizing the collected human locomotion data as the reference, an optimization problem is employed to design a stable and optimal gait which can be directly implemented on the specific robot or test subject.

\subsection{Motion Capture with IMU}

There have been many methods proposed for ambulatory measurement of human joint angles. In particular, Luinge 
and Veltink [17] proposed a Kalman filter which integrates the 3D angular velocity while applying heading corrections based on accelerometer readings. This approach is prone to integration drift of the gyroscope for systems which need to operate for long durations of time such as prostheses. A more advanced kinematic filtering method was proposed by Roetenberg et al. [18] for the XSens MVN motion capture suit. This approach uses a kinematic model of the individual body segments which is used to update a Kalman filter and provide the positions of each joint and segment of the body. Motion capture systems have also been shown to be effective for robotic teleoperation such as the method proposed by Miller et al. [19] in which an inertial motion capture system was successfully used to teleoperate the NASA Robonaut. This system used a complementary filter to fuse accelerometers, gyroscopes, and magnetometers to estimate poses which were then used to compute an inverse kinematic relationship for pose recreation on the robot.

The algorithm used for motion capture in this work is a planar modification of the model-based EKF first presented by Šlajpah et al. [20]. In this approach, the human extremities are modeled as a kinematic chain built from a location of negligible acceleration. The concept is based on a kinematic relation similar to a series of inverted pendula, where the acceleration of any point $\mathrm{B}$ on a rigid body can be determined if the angular velocity, angular acceleration and linear acceleration of other point $\mathrm{A}$ on the body are known through the relation:

$a_{B}=a_{A}+\omega \times\left(\omega \times r_{A B}\right)+\dot{\omega} \times r_{A B}$,

where $r_{A B}$ is the distance from point $\mathrm{A}$ to point $\mathrm{B}$ and $\omega$ is the angular velocity of the link. The algorithm used in this work is different in two aspects: the kinematic model of the human legs is assumed to be composed of joints with ranges of motion limited to flexion/extension, and the kinematic chain is built from the hip. Since AMPRO has restricted actuation in solely the saggital plane and because joint variations in the coronal plane are not used in the proposed control approach, only measurements resulting in joint flexion and extension are used in the model update. To express the segment estimation in terms of the available joint mobility of AMPRO, the measurements from the IMUs are projected onto the saggital plane at each time step before they are passed to the filter. Additionally, we assume that the forward velocity of the hip is constant [21], [22] and that sinusoidal movement of the hip in the vertical direction will yield negligible acceleration in comparison to walking dynamics.

An EKF is instantiated for each segment in the model and updated sequentially along the kinematic chain from the hip. More specifically, the hip joint is instantiated as a fixed-base where the base acceleration and angular velocity are zero. Each distal joint in the chain is then treated as a moving base, where the base acceleration and angular
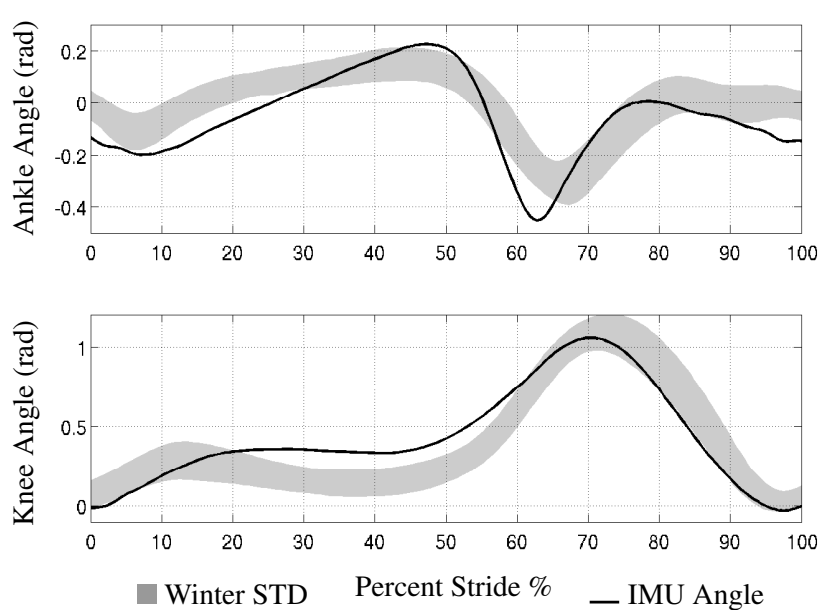

Fig. 2: Average joint angles for human subject using EKF filter as compared to Winter [23]. The trajectories are used as a comparison to show that the subject is walking with qualitatively humanlike trajectories for use in the trajectory optimization.

velocity are provided by the previous link. Each EKF update estimates the states $x_{k}=\left[\omega^{S}, \dot{\omega}^{S}, q^{E}, \dot{q}^{E}\right]^{T}$, with $\dot{\omega}^{S}$ the first time derivative of the angular velocity in the segment (S) frame and $\dot{q}^{E}$ the first time derivative of the earthframe (E) quaternion. The measurements for each link are $z_{k}=\left[\omega_{k}^{S}, \dot{\omega}_{k}^{S}, a_{k}^{S}\right]$ with $a_{k}^{S}$ the linear acceleration of the link, which is the primary measurement coupled to the previous link. Specifically, the acceleration from the previous joint is used in the estimation model as (1) where $a_{B}$ is the expected accelerometer reading, and $a_{A}$ is passed from the previous link. The estimation then proceeds through each joint according to the method detailed in [20].

Finally, the estimated orientations from the multibody EKFs are then used to extract the joint configuration using each link's quaternion attitude in the global frame ${ }^{E} q$, from which the joint rotations used in the overall model $q_{j}$ can be found as:

$q_{j}=q_{P}^{*} \otimes q_{D}$,

where $q_{P}^{*}$ denotes the quaternion conjugate, $\otimes$ is the quaternion product, $q_{D}$ is the distal segment orientation and $q_{P}$ is the proximal segment orientation.

During the experiment, the subject was asked to walk along a straight line for several steps while wearing seven IMUs to capture the walking behavior of the feet, shanks, thighs, and torso. The joint states are estimated and collected with the EKF algorithm. Then the joint angles are calculated by converting the joint rotation found in (2) to an Euler angle representation. Finally, several steps are averaged to yield their unique trajectories for optimization. While a thorough investigation on the accuracy of the 3D estimation method was performed by Šlajpah et al., a comparison of the walking captured via the $2 \mathrm{D}$ projection was not validated with 


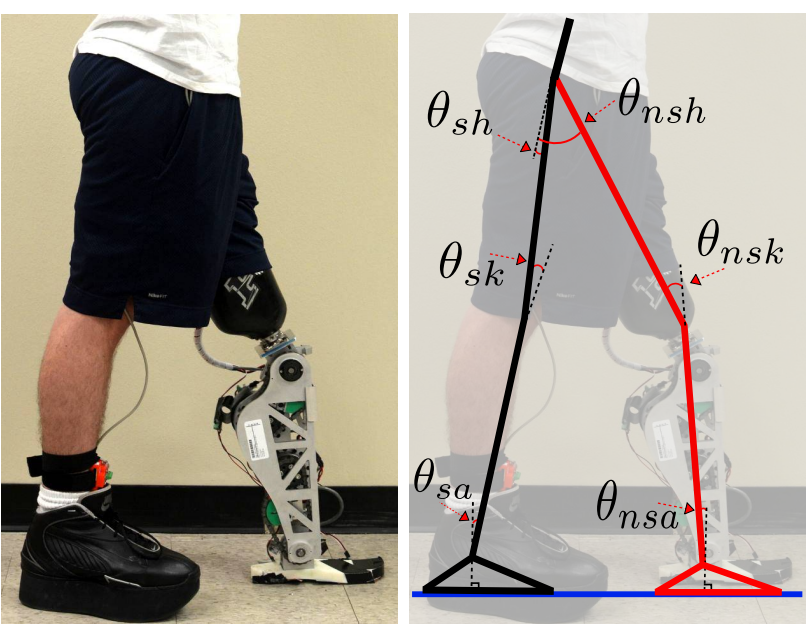

Fig. 3: Amputee subject with AMPRO (left) and the robotic model (right).

an optical tracking system. To verify that the EKF method is qualitatively capturing the human motion, knee and ankle joint angles of a healthy subject are compared to a standard set of saggital plane gait kinematics findings by Winter [23] as shown in Fig. 2.

\subsection{Robot Model.}

A 7-link planar bipedal robot (one torso, two thighs, two calves and two feet) with anthropomorphic parameters corresponding to the test subject is considered as the robotic model of a human in this work. Due to the existence of discrete behavior present in walking, i.e., impacts due to foot strike, we represent the bipedal robot as a hybrid system [24] with the configuration space $Q_{R}: \theta=\left(\theta_{s a}, \theta_{s k}, \theta_{s h}, \theta_{n s h}, \theta_{n s k}\right.$, $\left.\theta_{n s a}\right)^{T}$ as shown in Fig. 3. The equations of motion of the continuous dynamics are derived using the Euler-Lagrange formula:

$D(\theta) \ddot{\theta}+H(\theta, \dot{\theta})=B u$,

where $D(\theta) \in \mathbb{R}^{6 \times 6}$ is the inertial matrix and $H(\theta, \dot{\theta}) \in$ $\mathbb{R}^{6 \times 1}$ contains the terms resulting from the Coriolis effect $C(\theta, \dot{\theta}) \dot{\theta}$ and the gravity vector $G(\theta)$. The torque map $B=$ $I_{6}$ and $u$ is the vector of torque inputs. With the notation $x=(\theta ; \dot{\theta})$, the affine control system $\dot{x}=f(x)+g(x) u$ can be obtained by reformulating (3) [25]. The discrete behavior of impact is modeled with the perfectly plastic impact assumption; more details can be found in [26-28]. test test

Human-Inspired Outputs. With the goal of characterizing human walking, we take the perspective of viewing the "complex" human locomotion system as a "black box". The goal becomes to drive the actual robot outputs $y^{a}(\theta)$ to the desired human outputs $y^{d}(t, \alpha)$ that are found can be represented by the canonical walking function (CWF) with a parameter set $\alpha$ in the formula as:

$y_{c w f}(t, \alpha)=e^{-\alpha_{4} t}\left(\alpha_{1} \cos \left(\alpha_{2} t\right)+\alpha_{3} \sin \left(\alpha_{2} t\right)\right)+\alpha_{5}$.

This function is shown to be able to characterize human motion primitives universally including walking, running and stair climbing in previous work [26,21]. For the pinned 7link bipedal robot model considered in this paper, a total of 6 outputs are of interest including forward hip velocity (hip), knee angles ( $s k, n s k)$, non-stance slope ( $n s l)$, torso angle (tor) and non-stance foot angle ( $n s f)$; for details, refer to [29]. Therefore, we introduce the human-inspired outputs:

$y(\theta, \dot{\theta}, \alpha)=\left[\begin{array}{c}y_{1}(\theta, \dot{\theta}, \alpha) \\ y_{2}(\theta, \alpha)\end{array}\right]=\left[\begin{array}{c}y_{1}^{a}(\theta, \dot{\theta})-v_{h i p} \\ y_{2}^{a}(\theta)-y_{2}^{d}(\rho(\theta), \alpha)\end{array}\right]$,

where $y_{1}(\theta, \dot{\theta})=y_{1}^{a}(\theta, \dot{\theta})-v_{\text {hip }}$ is the difference between the actual and desired hip velocity, and relative degree two outputs $y_{2}(\theta, \alpha)=y_{2}^{a}(\theta)-y_{2}^{d}(\rho(\theta), \alpha)$ contain the differences between the actual and desired relative degree two outputs. Note that, the desired relative degree two outputs are defined as $y_{2}^{d}(\rho(\theta), \alpha)=\left[y_{c w f}\left(\rho(\theta), \alpha_{i}\right)\right]_{i \in O}$ with $\alpha_{i}=$ $\left(\alpha_{i, 1}, \alpha_{i, 2}, \alpha_{i, 3}, \alpha_{i, 4}, \alpha_{i, 5}\right)$ in (4), and $O=\{s k, n s k, n s l$, tor, $n s f$ \} is the set of relative degree two outputs. The parameters of all of the outputs are then combined to yield a single vector $\alpha=\left(v_{\text {hip }}, \alpha_{s k}, \alpha_{n s k}, \alpha_{n s l}, \alpha_{t o r}, \alpha_{n s f}\right) \in \mathbb{R}^{26}$.

Upon observation of human locomotion data, the linearized forward hip position, $\delta p_{\text {hip }}(\theta)$, was discovered to increase linearly through the progress of a step cycle [21], [22], [30], therefore, motivating the phase variable:

$\rho(\theta)=\left(\delta p_{\text {hip }}(\theta)-\delta p_{\text {hip }}^{+}\right) / v_{\text {hip }}$,

which is therefore used to parameterize a given walking gait as indicated in the formula of the desired outputs. Note that, the initial forward hip position $\delta p_{\text {hip }}^{+}(\theta)$ will be decided through the optimized gait which will be discussed later.

\subsection{Human-Inspired Optimization}

Using these outputs, the feedback linearization human-inspired controller [26] can be utilized to drive both $y_{1} \rightarrow 0$ and $y_{2} \rightarrow 0$ in a provably exponentially stable fashion. However, the robot will be "thrown-off" the designed trajectory when impacts occur. This motivates the introduction of the partial hybrid zero dynamics (PHZD) constraints aiming at yielding a parameter set $\alpha$ that ensures the tracking of relative degree two outputs remain invariant even through impacts, therefore guarantees stability of the walking gait. In particular, the partial zero dynamics (PZD) surface is defined as:

$\mathbf{P Z}_{\alpha}=\left\{(\theta, \dot{\theta}) \in T Q_{R}: y_{2}(\theta, \alpha)=\mathbf{0}, L_{f} y_{2}(\theta, \alpha)=\mathbf{0}\right\}$,

and the PHZD constraints can be explicitly stated as:

$\Delta_{R}\left(S_{R} \cap \mathbf{P Z} \mathbf{Z}_{\alpha}\right) \subseteq \mathbf{P} \mathbf{Z}_{\alpha}$,

(PHZD) 
where $\Delta_{R}$ and $S_{R}$ are the reset map and switching surface of the robot model, respectively. The detailed explanation of these constraints can be found in [26].

By enforcing the PHZD constraints discussed above, the human-inspired optimization can be used to generate robot or prosthetic trajectories that are both provably stable and human-like [26]. More importantly, for a lower-limb prosthesis interacting with humans in a safety critical fashion, more attention must be placed on the physical constraints that relate to safety and energy conservation. One particular goal, for example, is to optimize the torque profile such that it is within the limit of the motors in order to bear the human weight during the stance phase. Walking gaits which require optimal torques also tend to decrease the energy consumption of the device, prolonging battery life. These specifications yield the optimization problem subject to the PHZD and physical constraints as stated in the following:

$$
\alpha^{*}=\underset{\alpha \in \mathbb{R}^{26}}{\operatorname{argmin}} \operatorname{Cost}_{\mathrm{HD}}(\alpha)
$$

\section{s.t PHZD Constraints,}

Physical Constraints,

where the cost function (HIO) is the least-square-fit error between the human experimental data (discussed in Sec. 2.1) and the CWF representations (5). The explicit expression of the cost function is stated as follows:

$\operatorname{Cost}_{\mathrm{HD}}(\alpha)=\sum_{i \in O} \sum_{k=1}^{K_{i}}\left(y_{i}^{a}[k]-y_{i}^{d}\left(t_{i}^{a}[k], \alpha_{i}\right)\right)^{2}$,

where $t_{i}^{a}$ and $K_{i}$ are the discrete time and the number of discrete points for the recorded output $i \in O$, respectively. $O$ is the output set, which is defined based on the model.

Note that the physical constraints include additional constraints that ensure foot clearance and that the movement range of the joints is within hardware limits. The direct result of this optimization problem is the parameter set $\alpha$ that renders an optimal (w.r.t. torque, foot clearance, joint position and velocity) and provably stable human-like walking gait. Additionally, the gait can be directly implemented on the robot or prosthetic device.

To summarize, this optimization problem uses the trajectory of a healthy subject as the reference, which is subject to PHZD constraints (to ensure smooth transitions between stance and swing phase) and physical constraints (torque and angle limitations) such that the output gait is applicable for implementation on the prosthetic device. Therefore, the main advantages of utilizing this optimization problem are twofold: a) an optimal smooth gait can be designed for a specific amputee without hand tuning and, b) the output gait can be practically used directly on the prosthetic device with optimal torque and energy efficiency.

Remark. The term of stability mentioned here is specific to the mathematical stability of the designed gait, i.e., the gait has a limit cycle that is exponentially stable [24]. While this stability property does not guarantee stable walking (w.r.t the walking balance) of the prosthetic device, it plays a key role during the nonlinear prosthetic controller development. To be more specific, the main goal of this research is to translate robotic walking into the design and control of the prosthesis. A mathematically stable gait is of essential importance for realization of robotic walking for bipedal robots in both simulation and experiment. Utilizing this stable robotic walking the nonlinear controller, therefore, can be verified and improved on the robots before implementing on the prosthetic device.

\subsection{Prosthetic Trajectory Reconstruction}

The result of the optimization problem (HIO) is the parameter set $\alpha$ that define the human-inspired outputs. Via these outputs, we can obtain desired joint angles and angular velocities for the robot in every iteration through the inverse projection from the PHZD surface. This is achieved through a methodology termed the $P H Z D$ reconstruction [31]. In particular, on the PHZD surface, the zero dynamic coordinates can be defined as:

$\xi_{1}=\delta p_{h i p}(\theta):=c \theta$,

$\xi_{2}=y_{1}^{a}(\theta, \dot{\theta}):=\delta \dot{p}_{h i p}(\theta):=c \dot{\theta}$,

where $c$ is the coefficient array defining the linearized hip position $\delta p_{\text {hip }}(\theta)$ [29]. As the direct result of (6) and (9), the desired relative degree two outputs can be stated as $y_{2}^{d}(\rho(\theta)$, $\alpha)=y_{2}^{d}\left(\xi_{1}, \alpha\right)$. Similarly, due to the linearity of the actual relative degree two outputs, we have $y_{2}^{a}=H \theta$ and $\dot{y}_{2}^{a}=H \dot{\theta}$. Therefore, utilizing the fact that the actual outputs are equal to the desired outputs on the PHZD surface, we have the following relationships between the desired joints states and the desired outputs of the robot, which are given by:

$$
\begin{gathered}
\theta_{d}(\xi)=\Psi\left(\xi_{1}, \alpha\right)=\left[\begin{array}{c}
c \\
H
\end{array}\right]^{-1}\left(\begin{array}{c}
\xi_{1} \\
y_{2}^{d}\left(\xi_{1}, \alpha\right)
\end{array}\right), \\
\dot{\theta}_{d}(\xi)=\Phi\left(\xi_{1}, \xi_{2}, \alpha\right)=\left[\begin{array}{c}
c \\
H
\end{array}\right]^{-1}\left(\begin{array}{c}
v_{h i p} \\
\frac{\partial y_{2}^{d}\left(\xi_{1}, \alpha\right)}{\partial \xi_{1}} \xi_{2}
\end{array}\right) .
\end{gathered}
$$

The immediate result of this expression is that by knowing $\xi_{1}$ and $\xi_{2}$ (which are the linearized forward hip position and forward hip velocity, respectively), the desired angles and velocities can be obtained directly using the parameter $\alpha$. More importantly, the resulting joint angles and velocities are guaranteed to be smooth, human-like and optimal. 


\section{PROSTHETIC CONTROLLER DESIGN}

This section will begin by briefly introducing the framework of variable impedance control. This traditional control approach is utilized in the development of a novel control Lyapunov function (CLF) [32] based model independent quadratic program (MIQP) controller for prosthetic joints.

\subsection{Impedance Control for Prosthesis}

Based on the notion of impedance control in [33], the torque at each joint during a single step can be represented in a piecewise fashion by a series of passive impedance functions [10] of the form:

$\tau^{i m p}=k\left(\theta-q^{e}\right)+b \dot{\theta}$,

where, $k, q^{e}$ and $b$ represent the impedance parameters for stiffness, equilibrium angle and damping, respectively, which are constant for a specific phase.

While impedance control with a finite state machine is one of the most widely used algorithms suggested to date $[13,10]$, one main challenge of impedance control is that it requires the choice of the control parameters for each phase. Currently, clinicians and prosthetic researchers often choose these parameters by trial and error hand tuning for each patient as noted in [10]. Motivated by this shortcoming, the authors took a different approach in the previous work [34] to learn the impedance parameters for a lower-limb prosthesis by the observation of unimpaired human walkers. The results have been validated in both simulation and experiment with a transfemoral prosthetic device. As an extension of this work, it is also shown that the impedance parameters can be estimated by using the least-square-error method to fit the simulated data or experimental data with the piecewise impedance functions. Therefore, by using the impedance estimation algorithm as discussed in the previous works, the impedance controller can be implemented directly on the robot or prosthetic device with minimum tuning $[15,16]$.

\subsection{CLF Model Independent QP}

As a means for stabilizing systems undergoing impacts, rapidly exponentially stabilizing control Lyapunov functions (RES-CLFs) were introduced in [32] to yield controllers with stronger convergence guarantees. Quadratic programs can be used to realize RES-CLFs via inequality constraints. When combined with impedance control (implemented as a feed -forward term), the result is a novel feedback control methodology: Model Independent Quadratic Programs (MIQP)+Impedance control.

\subsubsection{Human-Inspired Control Revisited}

With the human-inspired outputs defined in (5), the dynamics in (3) can be reformulated as:

$\left[\begin{array}{c}\dot{y}_{1} \\ \ddot{y}_{2}\end{array}\right]=\underbrace{\left[\begin{array}{c}L_{f} y_{1}(\theta, \dot{\theta}) \\ L_{f}^{2} y_{2}(\theta, \dot{\theta})\end{array}\right]}_{L_{f}}+\underbrace{\left[\begin{array}{c}L_{g} y_{1}(\theta, \dot{\theta}) \\ L_{g} L_{f} y_{2}(\theta, \dot{\theta})\end{array}\right]}_{A} u$,

where $L_{f}$ is the Lie derivative and $A$ is the dynamic decoupling matrix, which is invertible because of the specific criterion of the outputs selection [16]. By picking $u=A^{-1}\left(-L_{f}\right.$ $+\mu)$, equation (13) becomes:

$\left[\begin{array}{l}\dot{y}_{1} \\ \ddot{y}_{2}\end{array}\right]=\mu$

By designing $\mu$ properly (see [26]) one can drive both $y_{1} \rightarrow$ 0 and $y_{2} \rightarrow 0$ exponentially. However, due to the lack of the model information, it is not possible to realize this controller on prostheses. As a result, traditional PID control or variable impedance control are typically seen as a more favorable option-it does not require accurate model information, i.e., it is model independent. However, PID controllers lack formal guarantees (when applied to nonlinear systems) and require hand tuning [35]. This motivates the need to find a new control strategy that overcomes the weaknesses of PID control while maintaining model insensitivity.

\subsubsection{CLF MIQP}

By defining the vector $\eta=(y, \dot{y}) \in \mathbb{R}^{n_{1}+2 \times n_{2}}$ with $n_{1}, n_{2}$ denoting the numbers of relative degree one outputs and relative degree two outputs, respectively, equation (14) can be written as a linear affine control system:

$\dot{\eta}=\underbrace{\left[\begin{array}{c}0_{n_{1} \times n_{1}} 0_{n_{1} \times n_{2}} 0_{n_{1} \times n_{2}} \\ 0_{n_{2} \times n_{1}} 0_{n_{2} \times n_{2}} I_{n_{2} \times n_{2}} \\ 0_{n_{2} \times n_{1}} 0_{n_{2} \times n_{2}} 0_{n_{2} \times n_{2}}\end{array}\right]}_{F} \eta+\underbrace{\left[\begin{array}{c}I_{n_{1} \times n_{1}} 0_{n_{1} \times n_{2}} \\ 0_{n_{2} \times n_{1}} 0_{n_{2} \times n_{2}} \\ 0_{n_{2} \times n_{1}} I_{n_{2} \times n_{2}}\end{array}\right]}_{G} \mu$.

Considering the Continuous Algebraic Riccati Equations (CARE) with $P=P^{T}>0$ :

$F^{T} P+P F-P G G^{T} P+I=0$,

we can obtain the optimal solution $\mu=-G^{T} P \eta$. The solutions of the CARE also allows for the construction of a rapidly exponentially stabilizing control Lyapunov function $(R E S-C L F)$ [36]. By defining $\eta_{\varepsilon}=\left(y_{p} / \varepsilon ; \dot{y}_{p}\right)$ with $\varepsilon>0$, we define the positive definite RES-CLF to be:

$V_{\varepsilon}(\eta)=\eta^{T}\left[\begin{array}{cc}\frac{1}{\varepsilon} I & 0 \\ 0 & I\end{array}\right] P\left[\begin{array}{cc}\frac{1}{\varepsilon} I & 0 \\ 0 & I\end{array}\right] \eta:=\eta^{T} P_{\varepsilon} \eta$.

Differentiating this function yields:

$\dot{V}_{\varepsilon}(\eta)=L_{F} V_{\varepsilon}(\eta)+L_{G} V_{\varepsilon}(\eta) \mu$ 
where $L_{F} V_{\varepsilon}(\eta)=\eta^{T}\left(F^{T} P_{\varepsilon}+P_{\varepsilon} F\right) \eta, L_{G} V_{\varepsilon}(\eta)=2 \eta^{T} P_{\varepsilon} G$.

In order to exponentially stabilize the system, we want to find $\mu$ such that, for a specifically chosen $\gamma>0$ [32], we have:

$L_{F} V_{\varepsilon}(\eta)+L_{G} V_{\varepsilon}(\eta) \mu \leq-\frac{\gamma}{\varepsilon} V_{\varepsilon}(\eta)$.

Therefore, an optimal $\mu$ could be found by solving the following quadratic program $(\mathrm{QP})$ :

$$
\begin{aligned}
m(\eta)=\underset{\mu \in \mathbb{R}^{n_{1}+n_{2}}}{\operatorname{argmin}} \mu^{T} \mu & \\
& \text { s.t } \quad \varphi_{0}(\eta)+\varphi_{1}(\eta) \mu \leq 0,
\end{aligned}
$$

where $\varphi_{0}(\eta)=L_{F} V_{\varepsilon}(\eta)+\frac{\gamma}{\varepsilon} V_{\varepsilon}(\eta)$ and $\varphi_{1}(\eta)=L_{G} V_{\varepsilon}(\eta)$. The end result of solving the QP problem is a piecewise local optimal control input $\mu$ which is independent of model information, i.e., we obtain a MIQP. More explicitly, the main principle of the MIQP algorithm is to construct a new linear control system (15) that only focuses on the errors between the actual outputs and desired outputs, while not requiring any information about the original model. Note that, in order to obtain optimal torques that are also subject to other constraints (for example, torque bounds due to hardware limits), we relax the CLF constraints with a large penalty value $p>0$ [24]. In particular, we consider the MIQP:

$$
\begin{aligned}
\underset{\delta, \mu) \in \mathbb{R}^{n_{1}+n_{2}+1}}{\operatorname{argmin}} p \delta^{2}+\mu^{T} \mu & \\
\text { s.t } \quad \varphi_{0}(\eta)+\varphi_{1}(\eta) \mu & \leq \delta, \\
\mu & \leq \mu_{M A X}, \\
-\mu & \leq \mu_{M A X} .
\end{aligned}
$$

This QP problem yields an optimal controller $\mu$ that regulates the errors of the output dynamics in a rapidly exponentially stable fashion while simultaneously guaranteeing the result input torque is physically applicable.

\subsection{MIQP+Impedance Control}

While MIQP control benefits from its model independent property in an optimal fashion, the controller will generate the same torque for two different systems if they have the same tracking error. Consequently, this will result the controller to be less responsive to the actual system and tend to have overshooting problem Therefore, model information is utilized in order to achieve a more responsive controller; this motivates the introduction of MIQP+Impedance control. With the impedance controller $\tau^{i m p}$ as a feed-forward term, the desired torque $\tau^{d}$ of the prosthetic joints can be stated as:

$\tau^{d}=\tau^{q p}+\tau^{i m p}$ where $\tau^{q p}$ is the torque computed from the MIQP problem. Taking this idea further, we add the impedance term $\tau^{i m p}$ into the MIQP construction for the total hardware torque bounds, which yields the following MIQP+Impedance formula as follows:

$$
\begin{array}{rlrl}
\underset{\left(\delta, \tau^{q p}\right) \in \mathbb{R}^{n_{1}+n_{2}+1}}{\operatorname{argmin}} p \delta^{2}+\tau^{q p T} \tau^{q p} & \\
\text { s.t } \varphi_{0}(\eta)+\varphi_{1}(\eta) \tau^{q p} \leq \delta-\varphi_{1}(\eta) \tau^{i m p}, & \text { (CLF) } \\
\tau^{q p} & \leq \tau_{M A X}^{q p}, & \text { (Max QP Torque) } \\
-\tau^{q p} & \leq \tau_{M A X}^{q p}, & \text { (Min QP Torque) } \\
\tau^{q p} & \leq \tau_{M A X}-\tau^{i m p}, & \text { (Max Input Torque) } \\
-\tau^{q p} & \leq \tau_{M A X}+\tau^{i m p} . & \text { (Min Input Torque) }
\end{array}
$$

By adding the impedance control as a feed-forward term into the input torque, the model independent dynamic system (15) gathers proper information about the system. It can, therefore, adjust $\tau^{q p}$ accordingly to accommodate for the feed-forward term to achieve good tracking. By setting the QP torque bounds $\tau_{M A X}^{q p}$, the overshoot problem will be eliminated. We also set the total input torque bounds for the QP problem such that the final optimal input torques (22) will satisfy the hardware torque bounds $\tau_{M A X}$ and allow the control to be implemented practically on the robots or prosthetic devices.

Remark. In order to make the MIQP controller work properly, there are three parameters to be determined, which are $\varepsilon, p$ and the torque bounds $\tau_{M A X}^{*}$ with $\tau_{M A X}^{*}=\max \left\{\tau_{M A X}^{q p}\right.$, $\left.\tau_{M A X}\right\}$. Specifically, $\varepsilon$ determines the outputs convergence rate; $p$ is the CLF penalty term that regulates the wellness of the tracking performance and $\tau_{M A X}^{*}$ is defined based on the hardware limitation. While $\tau_{M A X}^{*}$ is determined by the device, changing $\varepsilon$ and $p$ will affect the performance of the QP. For example, if $\varepsilon$ or $p$ are increased (corresponding to quicker convergence and heavier penalty for bad tracking), the tracking will be improved while at the same time requiring more control torque input. Consequently, the QP tends to fail more given the fixed $\tau_{M A X}^{q p}$. More detailed discussion about the feasibility of the QP can be referred to [37].

The unique merit of the MIQP controller is that it only takes the output error as the input and stabilizes the output dynamics in a rapidly exponentially convergent manner. The original system may vary, however, the MIQP controller will adopt to different systems by increasing the calculated torque as long as the $\tau_{M A X}^{q p}$ is set to be sufficient large. Details about the feasibility conditions of the QP problem can be referred to [37].

\section{VERIFICATION ON AMBER}

Before implementing the optimal controller on a prosthetic device with an amputee subject, the controller is verified on 

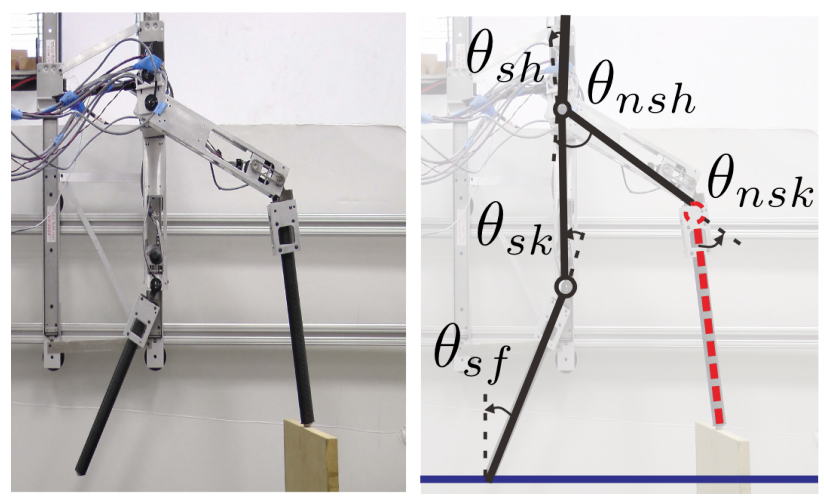

Fig. 4: The biped robot AMBER (left) and the angle conventions (right). The right leg with red dash line denotes the prosthetic device; the red dash circle represents the prosthesis joint that will be controlled using prosthetic controllers.

the bipedal robot AMBER in both simulation and experiment. The results are discussed in this section.

\subsection{AMBER Test Platform}

AMBER (short for A\&M Bipedal Experimental Robot) is a planar bipedal robot with 5 links (one torso, two thighs and two calves, see Fig. 4). With pointed feet configuration, AMBER is powered by 4 DC motors and is thus underactuated at the ankle. In previous work, AMBER has achieved stable and human-like walking experimentally using a voltage $\mathrm{P}$ controller [38]. In this work, we use AMBER as the platform to test the proposed prosthetic controller. The right calf is assumed to be the "prosthetic device" which has the same length and mass configuration of the left calf that is marked as the "healthy leg". The proposed controller will be implemented on the prosthetic device, i.e., on the right knee joint. The controller for the remaining actuators will still be the original voltage $\mathrm{P}$ controller as discussed in [38].

The configuration space $Q_{A}$ of the AMBER model is defined with coordinates given as: $\theta=\left(\theta_{s a}, \theta_{s k}, \theta_{s h}, \theta_{n s h}, \theta_{n s k}\right)^{T}$ as shown in Fig. 4. With the mass and length properties corresponding to the physical robot AMBER, the equations of motion for the robot can be obtained with equation (3). Note that, since AMBER has DC motors with small inductances, the electromechanical system with voltage inputs have the following form:

$V_{\text {in }}=R_{a} i_{a}+K_{\omega} \omega$

where $V_{\text {in }} \in \mathbb{R}^{4 \times 1}$ is the vector of voltage inputs to the motors, $i_{a} \in \mathbb{R}^{4 \times 1}$ is the vector of currents through the motors, $R_{a} \in \mathbb{R}^{4 \times 4}$ is the resistance matrix, and $\omega \in \mathbb{R}^{4 \times 1}$ is the vector of motor speed which has the relation as $\omega=r_{m} \dot{\theta}$ with $r_{m} \in \mathbb{R}^{4 \times 4}$ denoting the total reduction of the system. Since the motors are controlled individually, with the torque constant $K_{\varphi} \in \mathbb{R}^{4 \times 4}$, the applied inputs are:

$u=K_{\varphi} R_{a}^{-1}\left(V_{i n}-K_{\omega} \omega\right)$.

Therefore, the impedance torque and QP torque discussed in Sec. 3 will be replaced with impedance voltage $V^{i m p}$ and QP voltage $V^{q p}$, respectively [16].

\subsection{Simulation Verification with AMBER}

With the model in hand, the robotic gait can be obtained using the optimization problem as discussed in Sec. 2.2 with outputs specific to the model of AMBER [16]. The simulation results of AMBER is then discussed. The tracking results of the prosthesis joint by using different controllers will be compared. Robustness tests is also performed and compared with using different controllers.

\subsubsection{Tracking Performance with Different Controllers}

With the exception of the prosthesis joint, on which different controllers will be implemented, the remaining joints will be controlled with the voltage $\mathrm{P}$ control. Three different controllers are tested as the prosthetic controller: P control, impedance control and MIQP+Impedance control. Fig. 6 shows the tracking performances of the prosthesis knee joint using these three controllers. Using the tracking results of $P$ control as the nominal reference as shown in Fig. 6a, we can see that the MIQP+Impedance control improves the tracking performance for both stance and non-stance phases by more than 10 times w.r.t the $R M S$ error, while impedance control yields worse tracking results.

\subsubsection{Stability Testing}

Stability and robustness are another fundamental requirements for a prosthesis controller. Two robustness tests are

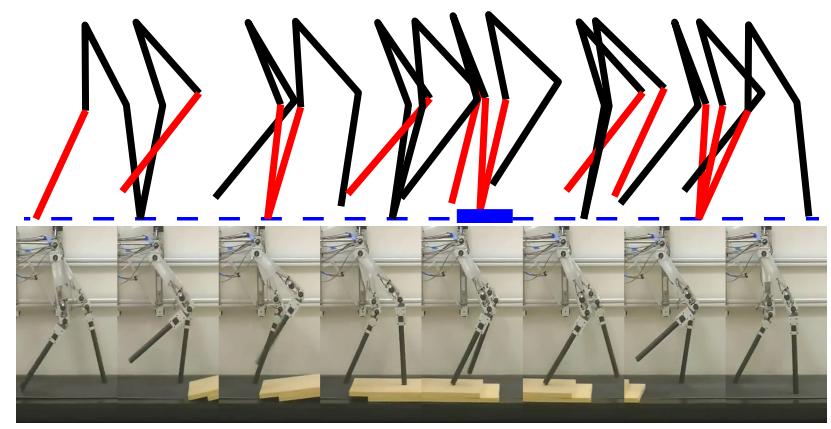

Fig. 5: Gait tiles of walking over an obstacle with $\mathrm{MIQP}+$ Impedance control in both simulation (top) and experiment (bottom). 


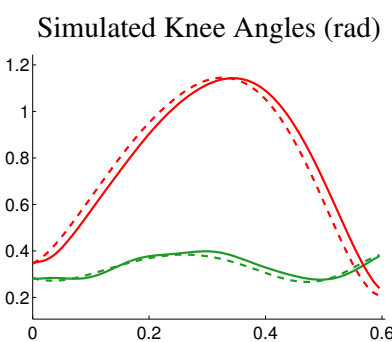

(a) Voltage control

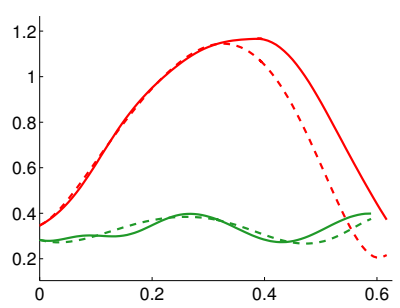

(c) Impedance control

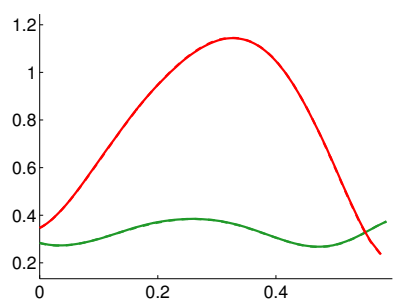

(e) MIQP+Imp control
Experiment Knee Angles (rad)

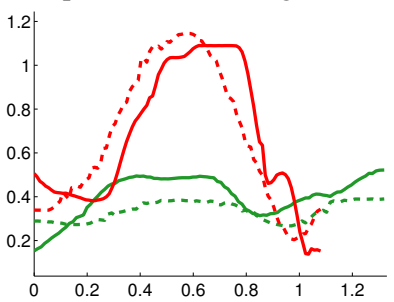

(b) Voltage control

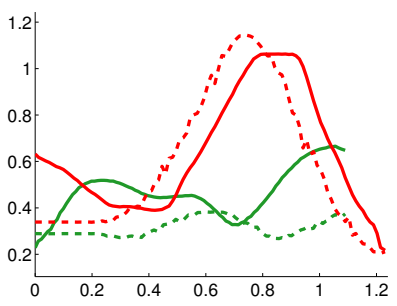

(d) Impedance control

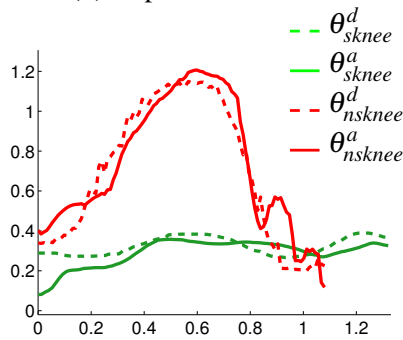

(f) MIQP+Imp control

Fig. 6: Actual and desired outputs of the prosthesis knee joint with different controllers in both simulation (left) and experiment (right).

applied to the robot in simulation; one is to add an instantaneous push and another one is to let the robot walk above an unforeseen obstacle. In particular, a $2 N$ impulse force (lasting for $0.05 s$ ) has been applied to the prosthetic leg while in swing phase. The results show that the prosthetic device with the proposed controller can tolerate this disturbance and maintain good tracking. We also tested the same disturbance using only the impedance controller; the tracking error becomes bigger due to the disturbance and the robot falls after 6 steps. For the obstacle test, we let the robot

Table 1: Simulation and Experiment Results Comparison of AMBER Using Different Controllers.

\begin{tabular}{|c|c|c|c|c|c|}
\hline \multirow{2}{*}{\multicolumn{2}{|c|}{ Control }} & \multicolumn{2}{|c|}{ Simulation } & \multicolumn{2}{|c|}{ Experiment } \\
\hline & & $e_{r m s}[\mathrm{rad}]$ & $e_{\max }[\mathrm{rad}]$ & $e_{r m s}[\mathrm{rad}]$ & $e_{\max }[\mathrm{rad}]$ \\
\hline \multirow{2}{*}{$\mathbf{P}$} & sknee & 0.0149 & 0.027 & 0.0863 & $0.1515^{*}$ \\
\hline & nsknee & 0.0523 & 0.0931 & 0.1538 & 0.3626 \\
\hline \multirow{2}{*}{ Imped } & sknee & 0.0258 & 0.0614 & 0.1902 & 0.3308 \\
\hline & nsknee & 0.1447 & 0.3115 & 0.1791 & $0.3517^{*}$ \\
\hline MIQP & sknee & $0.00098^{*}$ & $0.0022^{*}$ & $0.0602^{*}$ & 0.209 \\
\hline$+\operatorname{Imp}$ & nsknee & $0.0016^{*}$ & $0.0038^{*}$ & $0.1049^{*}$ & 0.3543 \\
\hline
\end{tabular}

walk over a $20 \mathrm{~mm}$ height obstacle. The gait tiles can be seen in Fig. 5, showing that the robot can overcome the obstacle smoothly. A similar test is also conducted with only impedance control. The robot can walk over the obstacle, however, the tracking performance becomes worse. More details can be found in [16].

\subsection{Experimental Verification on AMBER}

Starting with the estimated impedance parameters obtained from the simulation discussed above, we are able to tune the parameters within a small range and get sustainable walking by only using the impedance controller. The tracking result of using impedance controller can be seen in Fig. 6d. Compared to the tracking of $\mathrm{P}$ control as shown in Fig. $6 \mathrm{~b}$, the impedance control shows worse tracking performance.

MIQP + Impedance Control. Using the impedance parameters from the previous section, we apply the impedance control as the feed-forward term while using the MIQP as the feedback term to correct the tracking errors and reject the disturbances. From Fig. 6f, we can see the tracking with using MIQP+Impedance controller is the best among the three methods in both stance phase and non-stance phase (RMS error reduced by more than $50 \%$ for both phases). The detailed comparisons of both the simulation and experiment results are shown in Table. 1 . We also tested the robustness of the walking with MIQP+Impedance control, the robot was able to overcome a $40 \mathrm{~mm}$ block and could withstand big pushes on the prosthetic leg, while the impedance control can only overcome a $20 \mathrm{~mm}$ block. The experiment obstacle walking gait tiles are compared with the simulated gait tiles, as shown in Fig. 5.

With the verification in both simulation and experiment as discussed above, we can conclude that the proposed optimal nonlinear controller shows improved tracking performance and seems to be more robust to disturbances and obstacles than the traditional approaches.

\section{PROSTHETIC DEVICE IMPLEMENTATION}

With the systematic methodology including gait generation and controller implementation verified on the robot platform, we now have the framework to realize the real-time optimization based nonlinear controller experimentally on a selfcontained transfemoral prosthesis: AMPRO. The experiment setup including the design of the prosthesis AMPRO and the test subject is introduced first. Then the results of using the MIQP+Impedance controller along with other controllers are analyzed in a comparative study. 


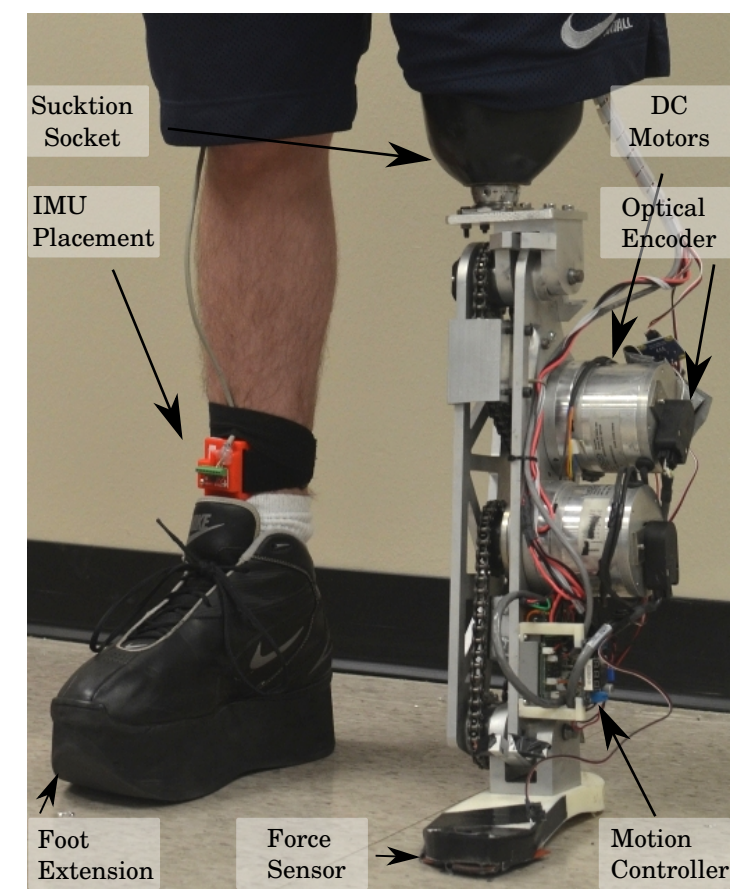

Fig. 7: Components diagram of the prosthesis AMPRO.

\subsection{Experiment Setup}

The experiment setup including the prosthetic device AMPRO and the amputee subject are introduced at this section. The two-level control architecture along with the IMU feedback sensing are also explained.

\subsubsection{AMPRO}

AMPRO (AMBER Prosthetic) is designed to be a high powered, compact and structurally safe device. The device uses a roller chain drive train consisting of a $374 \mathrm{~W}$ brushless DC motor (Moog BN34 silencer series) and a harmonic gearhead to actuate the ankle and knee joints in the sagittal plane. This design utilizes two incremental encoders for each motor and is designed to incorporate absolute encoders at the joints. Two Elmo motion controllers are used for low-level torque control purposes. Additionally, two FlexiForce (Parallax 30056) force sensors are located at the base of the foot (mounted at the toe and heel) to measure the normal reaction forces which are used for the purpose of leg switch. The prosthetic device is powered by a 8-cell LiPo battery with $4000 \mathrm{mAh}$ capacity. The technical diagram can be seen in Fig. 7 and the design specifications are listed in Table. 2.

\subsubsection{Test Subject}

The experimental subject during these experiments has been a unilateral amputee of three years due to osteosarcoma. The subject is a 19-year-old male that utilizes a passive knee

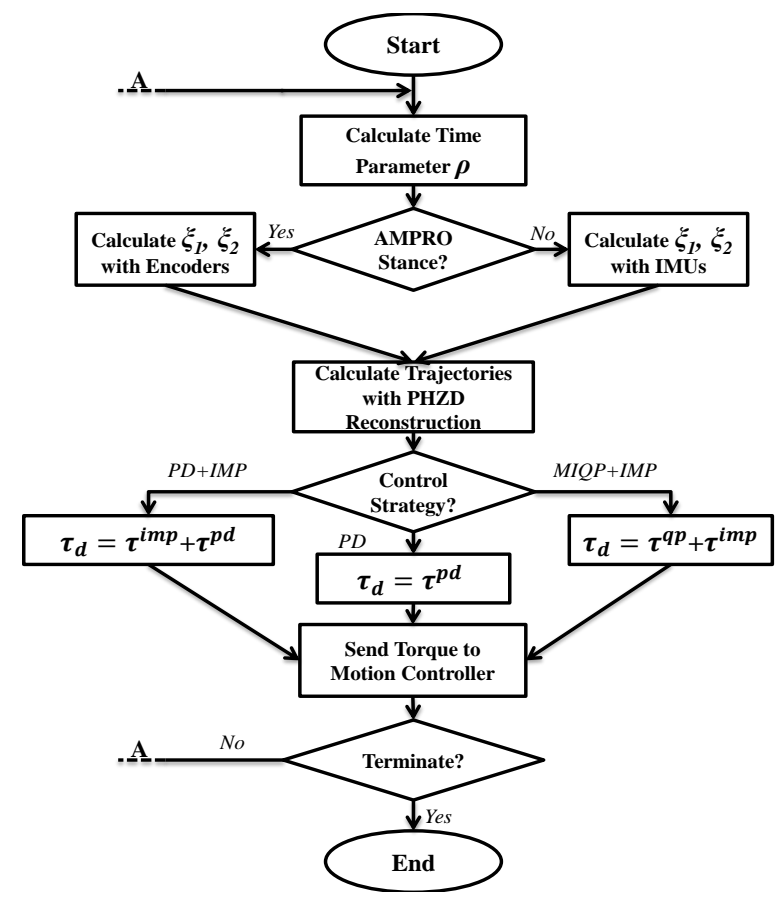

Fig. 8: Flow chart of the pseudo-code.

prosthesis (Ossur Total Knee 2100) for daily-use paired with a Ossur K2 Sensation foot. For the conducted experiments, the test subjects daily-use suction socket was connected to AMPRO using a standard pyramid connector. Some of the important parameters for the test subject were measured and are located in Table. 2. The residual limb of the subject was measured from the approximate location of the lateral condyle to the true ankle joint and from the true ankle joint to the base of the foot (for the calf and foot measurements respectively). During the experimental trials, the test subject used a custom made shoe that features a small extension of the sole of the shoe. This extension was required in order to have the subject's residual limb of equal length to the combination of AMPRO and the subject's suction socket connection.

Table 2: Specifications of the AMPRO and the Subject.

\begin{tabular}{|r|c|c|}
\hline Specifications & AMPRO & Amputee Subject \\
\hline Total Weight $(\mathrm{Kg})$ & 8.1 & 62 \\
\hline Total Height $(\mathrm{cm})$ & 56.3 & 173 \\
\hline Socket Length $(\mathrm{cm})$ & 8.8 & $*$ \\
\hline Calf Height $(\mathrm{cm})$ & 40.7 & 41.6 \\
\hline Foot Height $(\mathrm{cm})$ & 6.8 & 9 \\
\hline Shoe Extension $(\mathrm{cm})$ & $*$ & 5.7 \\
\hline Ankle Range of Movement $(\mathrm{deg})$ & $-20 \sim 30$ & $*$ \\
\hline Knee Range of Movement $(\mathrm{deg})$ & $0 \sim 70$ & $*$ \\
\hline Max Joint Velocity $(\mathrm{rpm})$ & 81.25 & $*$ \\
\hline
\end{tabular}




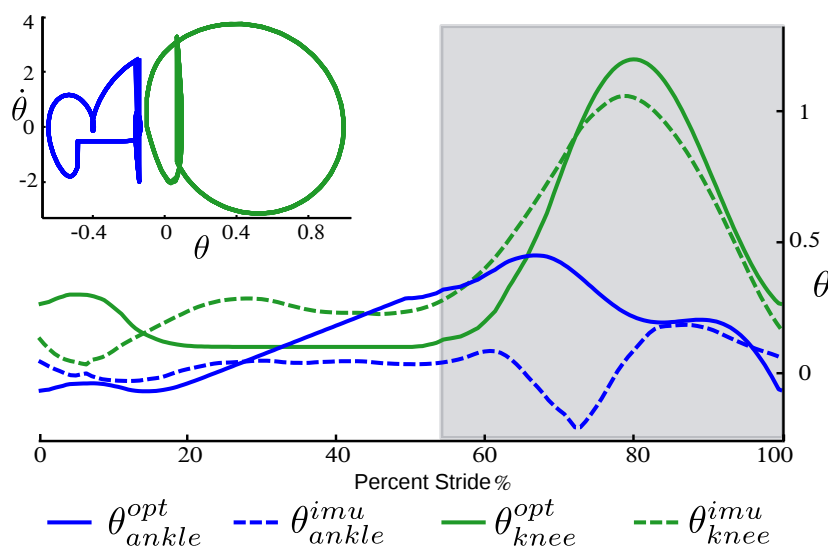

Fig. 9: Limit cycles for both the ankle and knee joints of flatfooted walking (upper left) and the comparison between the collected healthy human flat-foot walking data (IMU) and the joint angles optimized via (HIO); the shadowed region represents the swing phase.

\subsubsection{Control Architecture}

The architecture of control implementation consists a highlevel controller and a low-level controller. The high-level controller of AMPRO is coded into $\mathrm{C}++$ packages and run on the Robot Operating System (ROS). The complete code is realized independently with a low-power single-board computer: Beaglebone Black (BBB) at $200 \mathrm{~Hz}$. The pseudocode of the algorithm is shown as the flowchart in Fig. 8. To provide a point of human-robotic interaction, two IMUs are mounted on the shin and thigh of the human leg. As the IMU sensing is used only during human stance, an IMU on the human foot is eliminated based on the assumption that the user will walk with flat-foot during the interval of interest. The EKF internal model for each IMU is used to obtain relative orientation and velocity for both the knee and ankle. In particular, while the human leg is in stance, IMU readings are utilized to compute the forward hip position $\xi_{1}$ and forward hip velocity $\xi_{2}$; therefore, the desired swing trajectories of the prosthetic can be calculated accordingly using the PHZD reconstruction method discussed above. For hardware implementation, one $\mathrm{BBB}$ is dedicated to run the EKF algorithms as introduced in Sec. 2.2. The communication to the main $\mathrm{BBB}$ which runs the primary code structure is achieved over a networked crossover cable.

\subsection{Prosthetic Gait Generation}

An obvious problem encountered when designing a gait for an amputee is the lack of original locomotion data for the amputee. Human gait researchers and biomechanists have found that humans share a common pattern of joint trajectories during locomotion [23]. Therefore, a feasible approach is to use the nominal trajectories obtained from healthy subjects as the initial test gait for the amputee. While this is a common practice for prosthesis researchers and clinical physicians [10,39], this approach requires hand tuning and heuristic experience, which can be costly and time consuming. This motivates the authors in this work to take a different approach by using the human-inspired optimization problem to design an optimal gait for the amputee, therefore, eliminating the requirement of hand tuning.

In particular, the IMU motion capture system discussed in Sec. 2.2 is utilized to collect the flat-foot trajectory of a healthy subject who has the similar anthropomorphic parameters (limb lengths and body weight) of the amputee subject considered in this work. Using this trajectory set as the reference, and subject to both the PHZD constraints and physical constraints, the human-inspired optimization problem is leveraged to design a stable gait for the specific amputee subject. The limit cycle as shown in Fig. 9 indicates the stability of the gait mathematically. The optimized trajectories along with the IMU motion capture data are also shown in Fig. 9. We can see that the optimized knee angle follows a similar pattern as the healthy subject. However, the optimized ankle angle is different with the reference human trajectory especially in the late stance and swing phase. This mismatch is mainly due to the flat-foot walking assumption considered in this preliminary work. During the IMU data collection experiment, the healthy subject was unable to walk with strictly flat-foot. Therefore, there is always a minor foot roll at the end of the stance phase. Because of the flat-foot assumption, the swing foot is restricted to a small roll angle in order to have enough foot clearance. Consequently, the ankle angle dorsiflexes during the swing phase instead of plantar-flexing.

Remark. Flat-foot walking is a simplification of human walking. We realize that it introduces limitations on the prosthetic gait, however, it captures the essential behavior of walking that suits the capabilities of the first iteration of the prosthetic device AMPRO. Specifically, the first iteration of AMPRO is designed to walk with flat-foot, in order to test controllers obtained from the control framework designed for bipedal robots. More complex behaviors such as multicontact gaits will be addressed in the ongoing development of the next iteration of AMPRO which will also translate the control framework from the multi-contact capable bipedal robot, AMBER2.

\subsection{Experimental Results}

Before the implementation of MIQP+Impedance control on the prosthesis, the PD controller:

$\tau^{p d}=-K_{p}\left(\theta_{a}-\theta_{d}\left(\xi_{1}\right)\right)-K_{d}\left(\dot{\theta}_{a}-\dot{\theta}_{d}\left(\xi_{1}, \xi_{2}\right)\right)$, 


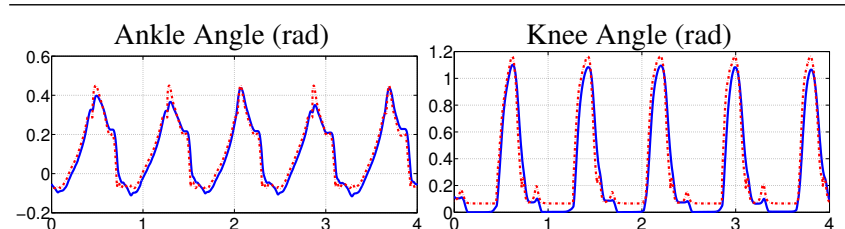

(a) PD control

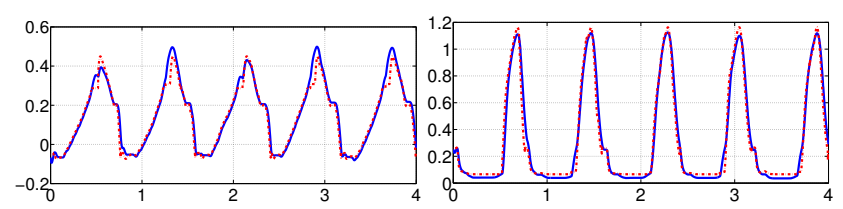

(b) PD+Impedance Control

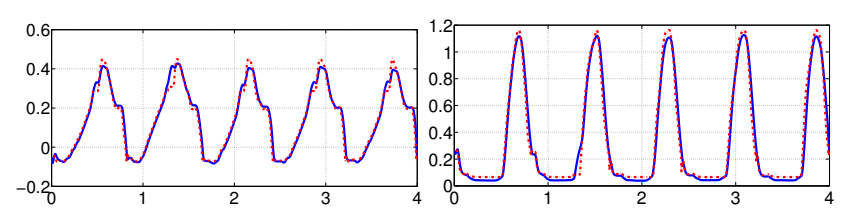

(c) MIQP+Impedance with high torque bounds

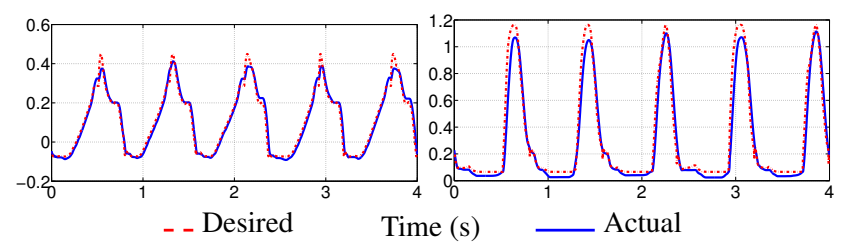

(d) MIQP+Impedance with low torque bounds

Fig. 10: Tracking comparison for different controllers.

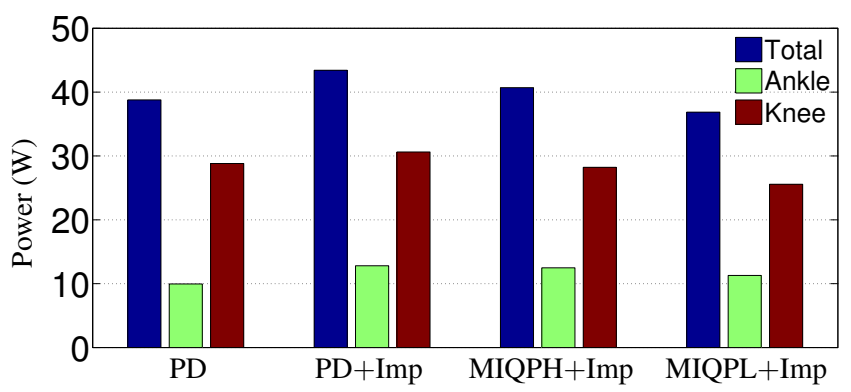

Fig. 11: Net mechanical power comparison of the prosthetic joints of one step (including stance phase and swing phase) with using different controllers.

is first realized to track the designed trajectories to achieve stable walking. The PD gains are tuned based on the feedback from the test subject. Walking trials were performed on a treadmill providing a constant speed of $1.3 \mathrm{mph}$. Utilizing the impedance estimation algorithm introduced in [40], the impedance parameters are then esitmated based on the experimental walking data obtained using PD control.

With the impedance parameters obtained in the previous section, we apply impedance control as the feed-forward term while using the MIQP control as the feedback term to track the desired joint trajectories. In particular, for the first round of test, we set both the torque bounds $\tau_{M A X}^{q p}$ and $\tau_{M A X}$ to be $40 \mathrm{Nm}$ which is determined based on the PD walking experiment data. In order to show the torque optimality of the proposed novel controller, the torque bounds $\tau_{\text {MAX }}^{q p}$ and $\tau_{M A X}$ are reduced to be $20 \mathrm{Nm}$ for the second round of test. While the novel control contains both the feedback term and feed-forward term, we also compare it with an augmented control strategy, PD+Impedance:

$\tau^{d}=\tau^{p d}+\tau^{i m p}$

which also includes the impedance control as a feed-forward term. The tracking results of using different controllers are shown in a comparative way in Fig. 10. The average mechanical power consumption of the prosthetic device during one step is shown in Fig. 11. More detailed performance comparisons are listed in the Table. 3. The gait tiles of the level walking using the proposed optimal controller along with the simulated gaits are shown in Fig. 12.

\subsection{Discussion}

With this controller, the tracking results of both the ankle and knee are plotted in Fig. 10 along with the results obtained through other controllers. It is evident that the tracking performance of both the ankle and the knee are exceptionally good for MIQP+Impedance control. The tracking results with lower torque bounds are shown in Fig. 10d. While the tracking performance is not as good as the tracking with higher torque bounds, it is better than using PD control. More importantly, this better tracking is achieved with lower torque and smaller total energy consumption when compared to PD control (5\% improvment), which can also be seen in Fig. 11 and Table. 3.

To illustrate the overall control performance more clearly, the experimental results (including tracking errors, maximum torque requirement and average net power consumption) of 5 steps are listed in Table. 3, thereby giving a detailed comparison. In particular, the best performances are highlighted in the table, from which we can see that except the max knee error (using PD+Impedance) and the minimum ankle power (using PD control), all of the best per-

Table 3: Experiment Results Comparison of AMPRO Using Different Controllers.

\begin{tabular}{c|cccccc}
\hline \multicolumn{2}{c}{ Control } & $e_{r m s}$ & $e_{\max }$ & $\sigma_{\text {std }}$ & $\tau_{\max }[\mathrm{Nm}]$ & $P[\mathrm{~W}]$ \\
\hline \multirow{2}{*}{ PD } & Ankle & 0.0377 & 0.1552 & 0.0185 & 22.456 & $9.9630^{*}$ \\
& Knee & 0.1096 & 0.3957 & $0.0363^{*}$ & 40.986 & 28.824 \\
\hline PD + & Ankle & 0.0356 & 0.1694 & 0.0165 & 23.787 & 12.805 \\
Imp & Knee & 0.0617 & 0.2875 & 0.0463 & 38.9207 & 30.627 \\
\hline MIQPL & Ankle & 0.0248 & $0.0965^{*}$ & 0.0216 & $19.846^{*}$ & 11.290 \\
+ Imp & Knee & 0.0906 & 0.3930 & 0.0399 & 37.083 & $25.577^{*}$ \\
\hline MIQPH & Ankle & $0.0246^{*}$ & 0.1066 & $0.0122^{*}$ & 20.944 & 12.482 \\
+ Imp & Knee & $0.0573^{*}$ & $0.2318^{*}$ & 0.0545 & $32.669^{*}$ & 28.229 \\
\hline
\end{tabular}




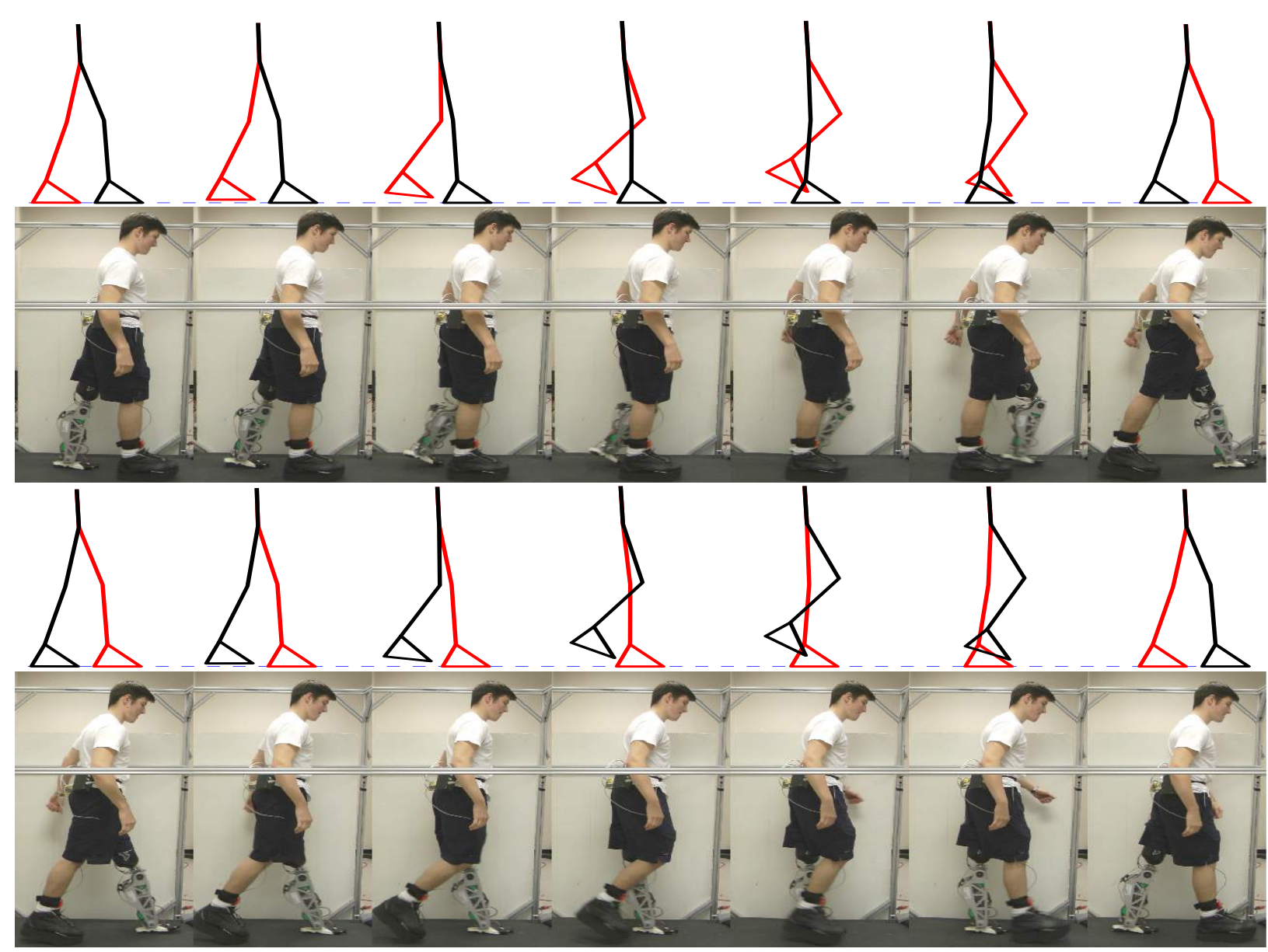

Fig. 12: Gait tile comparison between the outside NHS experimental walking and the simulated walking.

formances are achieved with the MIQP+Impedance controllers. Note that, we argue that the low power required of the ankle joint with PD control is because of the bad tracking performance (worst among all the controllers) of the PD controller. Therefore, to summarize, after comparing with different controllers, the MIQP+Impedance controller has the best balanced performance between tracking and power requirements.

The resulting powered prosthetic joint trajectories using the proposed optimal controller and the PD controller are compared with the IMUs collected healthy human locomotion data and the passive prosthetic walking data (the amputee walking with a passive device) in Fig. 13. Compared with the passive prosthetic walking, we can see that the ankle joint is improved for the power prosthetic walking with bigger movement. The human-likeness of the ankle joint will be improved in the future work with multi-contact walking. For the knee joint, we can see that both the passive device and powered device with different controllers have a similar swing pattern compared to the healthy human walking. However, for the stance knee trajectory, both the passive device and the powered device with PD control tend to lock the knee at the last portion of the stance phase. On the other hand, the powered device with MIQP+Impedance control has better performance with bigger knee bending and no knee locking. A slight delay was also noticed for the powered prosthetic walking when switching from stance phase to swing phase. This was caused by the delay of the force sensor recovering from the loaded status during the stance phase, which will be fixed by considering a load cell in the future design.

\section{CONCLUSIONS}

This work proposed a novel systematic methodology, including gait generation and optimal control, to achieve prosthetic walking for any specific subject, while at the same time aiming to improve control optimality and reduce clinical tuning. In particular, benefiting from the low-cost IMU motion capture system and the human-inspired optimization problem, a smooth and optimal prosthetic gait can be designed specifically for the amputee while simultaneously being applicable to the prosthetic device. The real-time optimization based nonlinear controller (MIQP+Impedance) 

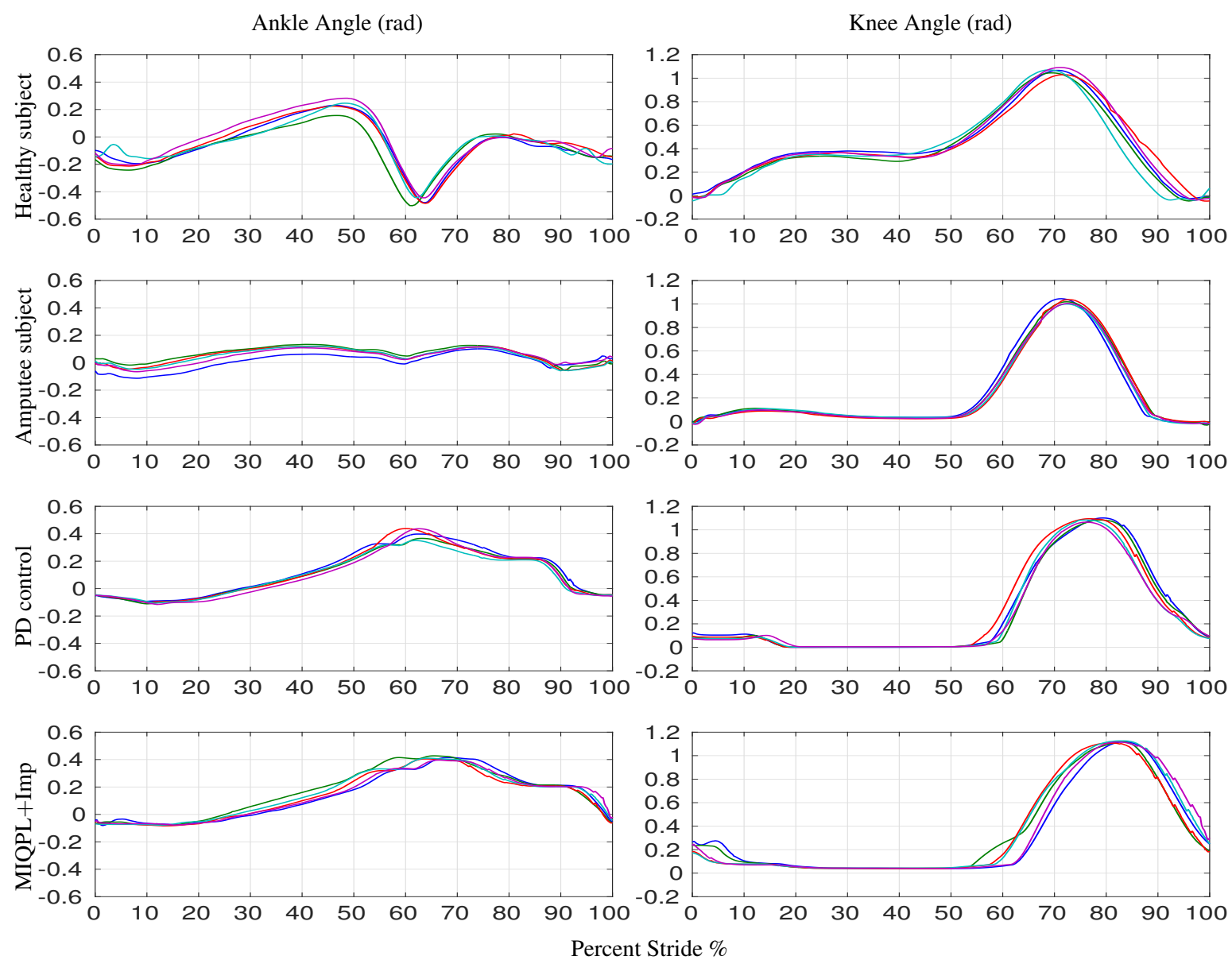

Fig. 13: Comparisons of both the ankle and knee joint angles of healthy human walking, passive prosthetic walking and powered prosthetic walking with PD control and MIQPL+Impedance control. Different colors represent different step trials.

shows improved performance with respect to both tracking and energy efficiency. This methodology has been first successfully validated on the bipedal robot platform, and then implemented on the custom built self-contained transfemoral prosthesis to achieve stable prosthetic walking with an amputee subject.

Traditional control approaches (e.g. variable impedance control) to powered prostheses rely on the extensive use of many control parameters in order to achieve successful operation of the device or for a particular subject. The methodology in this work takes the first steps toward designing the controllers and verifying their application both in the simulation and on the bipedal robot platform before implementation on a prosthetic device. Benefiting from this formal approach, it has the potential to reduce the clinical test and tuning for the amputee at the gait and control design stage. While future work should focus on showing that this approach also helps on the adaptation and comfortability of the amputee users, we believe that with the automation of the gait generation and controller design process proposed in this work, the tuning can potentially be reduced and the adaptation can be achieved in a more intuitive way. A new design of AMPRO is currently focused on reducing the size of the electric motors, optimizing the other structural components and adding load cells with the aim of providing a more comfortable experience for the user while reducing overall power consumption. Future work will be focused on the realization of a multi-contact walking gaits (as realized on bipedal robot AMBER2 in [29]) and different locomotion modes to achieve more natural and versatile human-like prosthetic walking.

\section{Acknowledgment}

This research is supported under: NSF CAREER Award CNS-0953823 and Texas Emerging Technology Fund 11062013. This research has approval from the Institutional Review Board from Texas A\&M University with IRB2014-0382F for testing with human subjects. 


\section{References}

1. T. Dillingham. Limb amputation and limb deficiency: Epidemiology and recent trends in the united states. Southern Medical Journal, 2002.

2. D. Winter. The Biomechanics and Motor Control of Human Gait: Normal, Elderly, and Pathological. University of Waterloo Press, 1991.

3. W. Flowers and Mann. Electrohydraulic knee-torque controller for a prosthesis simulator. ASME J. of Biomechanical Engineering, 99(4):3-8, 1977.

4. D. Grimes, W. Flowers, and M. Donath. Feasibility of an active control scheme for above knee prostheses. ASME J. of Biomechanical Engineering, 99(4):215-221, 1977.

5. J. Hitt, A. M. Oymagil, T. Sugar, K. Hollander, A. Boehler, and J. Fleeger. Dynamically controlled ankle-foot orthosis (dco) with regenerative kinetics: incrementally attaining user portability. In Robotics and Automation, 2007 IEEE International Conference on, pages 1541-1546.

6. A. M. Oymagil, J. K. Hitt, T. Sugar, and J. Fleeger. Control of a regenerative braking powered ankle foot orthosis. In Rehabilitation Robotics, 2007. IEEE 10th International Conference on, pages 28-34.

7. R. Rarick, H. Richter, A. van den Bogert, D. Simon, H. Warner, and T. Barto. Optimal design of a transfemoral prosthesis with energy storage and regeneration. In American Control Conference, pages 4108-4113, June 2014.

8. R. Gregg, T. Lenzi, L. Hargrove, and J. Sensinger. Virtual constraint control of a powered prosthetic leg: From simulation to experiments with transfemoral amputees. Robotics, IEEE Transactions on, 30(6):1455-1471, Dec 2014.

9. S. Au, M. Berniker, and H. Herr. Powered ankle-foot prosthesis to assist level-ground and stair-descent gaits. Neural Networks, 21(4):654 - 666, 2008.

10. F. Sup, A. Bohara, and M. Goldfarb. Design and Control of a Powered Transfemoral Prosthesis. The International journal of robotics research, 27(2):263-273, Feb. 2008.

11. A. Boehler, K. Hollander, T. Sugar, and D. Shin. Design, implementation and test results of a robust control method for a powered ankle foot orthosis (afo). In Robotics and Automation, 2008 IEEE International Conference on, pages 2025-2030.

12. K. Hollander and T. Sugar. A robust control concept for robotic ankle gait assistance. In Rehabilitation Robotics, 2007 IEEE 10th International Conference on, pages 119-123.

13. J. A. Blaya and H. Herr. Adaptive control of a variable-impedance ankle-foot orthosis to assist drop-foot gait. Neural Systems and Rehabilitation Engineering, IEEE Transactions on, 12(1):24-31, 2004.

14. N. Fey, A. Simon, A. Young, and L. Hargrove. Controlling knee swing initiation and ankle plantarflexion with an active prosthesis on level and inclined surfaces at variable walking speeds. Translational Engineering in Health and Medicine, IEEE Journal of, 2:1-12, 2014.

15. H. Zhao and A. D. Ames. Quadratic program based control of fully-actuated transfemoral prosthesis for flat-ground and up-slope locomotion. IEEE, American Control Conference, pages 4101 4107, 2014.

16. H. Zhao, S. Kolathaya, and A. D. Ames. Quadratic programming and impedance control for transfemoral prosthesis. Robotic and Automation, 2014 IEEE, International Conference on, pages 1341 $-1347$.

17. H. J. Luinge and P. H. Veltink. Measuring orientation of human body segments using miniature gyroscopes and accelerometers. Medical and Biological Engineering and computing, 43(2):273282, 2005

18. D. Roetenberg, H. Luinge, and P. Slycke. Xsens mvn: full 6dof human motion tracking using miniature inertial sensors. Xsens Motion Technologies BV, Tech. Rep, 2009.
19. N. Miller, O. C. Jenkins, M. Kallmann, and M. J. Mataric. Motion capture from inertial sensing for untethered humanoid teleoperation. In Humanoid Robots, 2004 IEEE/RAS 4th International Conference on, volume 2, pages 547-565.

20. S. Slajpah, R. Kamnik, and M. Munih. Kinematics based sensory fusion for wearable motion assessment in human walking. Computer methods and programs in biomedicine, 116:131-144, 2013.

21. H. Zhao, M. Powell, and A. D. Ames. Human-inspired motion primitives and transitions for bipedal robotic locomotion in diverse terrain. Optimal Control Applications and Methods, 35:730-755, 2013.

22. S. Jiang, S. Partrick, H. Zhao, and A. Ames. Outputs of human walking for bipedal robotic controller design. In American Control Conference (ACC), 2012, pages 4843-4848, 2012.

23. D. Winter. Biomechanics and Motor Control of Human Movement. Wiley-Interscience, New York, 2 edition, 1990.

24. A. Ames. Human-inspired control of bipedal walking robots. $A u$ tomatic Control, IEEE Transactions on, 59:1115 - 30, 2012.

25. S. Sastry. Nonlinear Systems: Analysis, Stability and Control. Springer, New York, 1999.

26. A. Ames. First steps toward automatically generating bipedal robotic walking from human data. Robotic Motion and Control, 422:89-116, 2012.

27. Y. Hürmüzlü and D. Marghitu. Rigid body collisions of planar kinematic chains with multiple contact points. Intl. J. of Robotics Research, 13(1):82-92, 1994.

28. E. Westervelt, J. Grizzle, C. Chevallereau, J. Choi, and B. Morris. Feedback Control of Dynamic Bipedal Robot Locomotion. CRC Press, 2007.

29. H. Zhao, W.-L. Ma, M. B. Zeagler, and A. D. Ames. Humaninspired multi-contact locomotion with amber2. In Cyber Physics System, 2014 IEEE International Conference on, pages 199-210.

30. A. Ames, R. Vasudevan, and R. Bajcsy. Human-data based cost of bipedal robotic walking. In Hybrid Systems: Computation and Control, Chicago, IL, 2011.

31. W.-L. Ma, H. Zhao, S. Kolathaya, and A. D. Ames. Humaninspired walking via unified pd and impedance control. In Robotic and Automation, 2014 IEEE International Conference on, pages $5088-5094$.

32. A. Ames, K. Galloway, and J. Grizzle. Control lyapunov functions and hybrid zero dynamics. In Decision and Control (CDC), 2012 IEEE 51st Annual Conference on, pages 6837-6842.

33. N. Hogan. Impedance control: An approach to manipulation. pages 304-313, 1984.

34. N. Aghasadeghi, H. Zhao, L. J. Hargrove, A. D. Ames, E. J. Perreault, and T. Bretl. Learning impedance controller parameters for lower-limb prostheses. Intelligent Robots and Systems, 2013 IEEE/RSJ International Conference on, pages 4268-4274.

35. D. Atherton and S. Majhi. Limitations of pid controllers. In American Control Conference, pages 3843-3847, 1999.

36. A. D. Ames, K. Galloway, J. Grizzle, and K. Sreenath. Rapidly exponentially stabilizing control lyapunov functions and hybrid zero dynamics. IEEE Trans. Automatic Control, 59:876-891, 2014.

37. B. Morris, M. Powell, and A. Ames. Sufficient conditions for the lipschitz continuity of qp-based multi-objective control of humanoid robots. In Decision and Control (CDC), 2013 IEEE 52nd Annual Conference on, pages 2920-2926.

38. S. N. Yadukumar, M. Pasupuleti, and A. Ames. Human-inspired underactuated bipedal robotic walking with amber on flat-ground, up-slope and uneven terrain. In Intelligent Robots and Systems, 2012 IEEE International Conference on, pages 2478-83, Portugal.

39. J. W. H. Herr and S. Au. Powered ankle-foot prosthesis. Biomechanics of the Lower Limb in Health, Disease and Rehabilitation, pages 72-74, 2007.

40. H. Zhao, J. Reher, J. Horn, V. Paredes, and A. D. Ames. Realization of nonlinear real-time optimization based controllers on selfcontained transfemoral prosthesis. Cyber Physics System, 2015 IEEE, International Conference on, pages 130-138. 